\title{
The Economic Shock of the Health Crisis in 2020: Comparing the Scale of Governments Support
}

\author{
Jacques Sapir* \\ EHESS-Paris, Foreign Member of the Russian Academy of Science, CEMI-IFAEE-Centre Robert de Sorbon, Paris, France \\ *e-mail: sapir@msh-paris.fr
}

Received September 13, 2021; revised September 15, 2021; accepted September 17, 2021

\begin{abstract}
Consequences of the COVID-19 epidemic have come as a major shock to all developed and developing economies. This occurred in a relatively short period of time. Even the collapse of 2008-2010, what was described as "subprime crises", seems to fade in comparison. This health crisis had seriously shaken the global economy and forced most governments to take measures to support economic activity that were considered exceptional at the time. These measures had moreover had significant consequences, either within emerging countries or within developed countries, and in particular in Europe. These consequences weighed on economic dynamics in the years that followed.
\end{abstract}

DOI: $10.1134 /$ S1075700721060186

The question of the extent of the reaction of governments to the economic consequences of the Covid19 pandemic arises with force. How have governments reacted? Have they given priority to budgetary action (subsidies) or have they given more priority to credit guarantees for financial actors and non-financial companies? Which governments have reacted the most and which have reacted the less? Is the scale of these reactions the product of political choices or ideological conceptions? These questions arise immediately.

Rulers, in France, but also of the United States and the United Kingdom have made statements on this subject, proclaiming that their administrations had reacted particularly well to the pandemic. We thus remember the "whatever it costs" pronounced in March 2020 by President Emmanuel Macron, ${ }^{1}$ which was repeated several times. ${ }^{2}$ From the results, still provisional, of the evolution of the economy in 2020, and from the estimate made by the International Monetary Fund of the amounts of aid, both budgetary and liquidity aid, we can make a first idea of the effort and reaction function of governments. We propose here a particular methodology to carry out this evaluation.

How to estimate governments effort? In a study published in 2020 predicting the possible impacts of the

\footnotetext{
${ }^{1}$ https://www.francetvinfo.fr/sante/maladie/coronavirus/quoiqu-il-en-coute-emmanuel-macron-lance-un-appel-general-ala-mobilisation-contre-le-coronavirus_3863731.html.

2 https://www.bfmtv.com/politique/elysee/emmanuel-macronce-quoi-qu-il-en-coute-je-l-assume VN-202012310209.html et https://www.lesechos.fr/economie-france/conjoncture/covidle-fmi-appuie-le-quoi-quil-en-coute-demmanuel-macron1261320.
}

current pandemic, one author uses the phrase "the mother of all recessions" to emphasize the magnitude of the shock and indirectly to qualify support measures [1]. There is no doubt that the crisis generated by the Covid-19 pandemic has reached historic dimensions [2]. Similarly, IMF projections and initial estimates from various national statistical institutes show a collapse in GDP growth rates that exceeds that of the 2008-2010 crisis. Here again, the available data point to an exceptionally brutal shock [3]. Since the advent of the pandemic, a growing number of publications have emerged to explain the consequences of this supply shock on demand, its potential persistence through stagnation traps [4], or the maintenance of containment measures, but also to qualify the extent of the support measures provided by the various governments [5].

It is assumed here that governments react to the emergence of a crisis situation, and react even more when the scale of the said crisis is important, for obvious economic reasons but also for political ones [6]. The Covid-19 pandemic has indeed constituted, by its economic consequences, a major disruption of economic activities. The measures taken to limit the spread of the virus, and in particular the containment or "lockdown" measures, have caused a recessionary shock, via supply and demand, of great magnitude and unparalleled in recent history [7]. It was both a supply shock, by the interruption of certain productions and demand by the fact that households were not able to consume normally. These shocks justified significant responses from governments, which have implemented more or less expansive fiscal policies [8], but 
Table 1. Presentation of data

\begin{tabular}{|c|c|c|c|c|}
\hline \multicolumn{5}{|c|}{ GDP percent } \\
\hline & $\begin{array}{c}\text { GDP Decrease } \\
\text { or increase for } 2020\end{array}$ & budget support & liquidity support & total support \\
\hline Austria & $-6.6 \%$ & $8.6 \%$ & $2.4 \%$ & $11.0 \%$ \\
\hline Belgium & $-6.4 \%$ & $7.2 \%$ & $11.9 \%$ & $19.1 \%$ \\
\hline Finland & $-2.9 \%$ & $3.0 \%$ & $7.0 \%$ & $10.0 \%$ \\
\hline France & $-8.2 \%$ & $7.7 \%$ & $15.8 \%$ & $23.5 \%$ \\
\hline Germany & $-4.9 \%$ & $11.0 \%$ & $27.8 \%$ & $38.9 \%$ \\
\hline Greece & $-8.2 \%$ & $11.0 \%$ & $4.9 \%$ & $15.9 \%$ \\
\hline Ireland & $2.5 \%$ & $5.4 \%$ & $1.5 \%$ & $6.9 \%$ \\
\hline Italy & $-8.9 \%$ & $6.8 \%$ & $35.5 \%$ & $42.3 \%$ \\
\hline Netherlands & $-3.8 \%$ & $4.5 \%$ & $8.3 \%$ & $12.8 \%$ \\
\hline Portugal & $-7.6 \%$ & $4.7 \%$ & $6.5 \%$ & $11.2 \%$ \\
\hline Slovakia & $-5.2 \%$ & $3.8 \%$ & $4.4 \%$ & $8.2 \%$ \\
\hline Slovenia & $-5.5 \%$ & $5.3 \%$ & $6.6 \%$ & $11.9 \%$ \\
\hline Spain & $-11.0 \%$ & $4.1 \%$ & $14.4 \%$ & $18.6 \%$ \\
\hline Canada & $-5.4 \%$ & $14.6 \%$ & $4.0 \%$ & $18.7 \%$ \\
\hline Japan & $-4.8 \%$ & $15.6 \%$ & $28.4 \%$ & $44.0 \%$ \\
\hline United Kingdom & $-9.9 \%$ & $16.3 \%$ & $16.1 \%$ & $32.4 \%$ \\
\hline United States & $-3.5 \%$ & $16.7 \%$ & $2.4 \%$ & $19.2 \%$ \\
\hline Russia & $-3.1 \%$ & $2.9 \%$ & $1.5 \%$ & $4.4 \%$ \\
\hline China & $2.3 \%$ & $4.7 \%$ & $1.3 \%$ & $6.0 \%$ \\
\hline India & $-8.0 \%$ & $3.1 \%$ & $5.1 \%$ & $8.1 \%$ \\
\hline Indonesia & $-2.1 \%$ & $2.7 \%$ & $0.9 \%$ & $3.5 \%$ \\
\hline Vietnam & $2.9 \%$ & $0.5 \%$ & $0.5 \%$ & $1.0 \%$ \\
\hline Australia & $-2.4 \%$ & $16.2 \%$ & $1.8 \%$ & $18.0 \%$ \\
\hline South Korea & $-1.0 \%$ & $3.4 \%$ & $10.2 \%$ & $13.6 \%$ \\
\hline Sweden & $-2.8 \%$ & $5.3 \%$ & $4.2 \%$ & $9.5 \%$ \\
\hline
\end{tabular}

Source. FMI, World Economic Outlook, April 2021 et Fiscal Monitor: Database of Country Fiscal Measures in Response to the COVID-19 Pandemic.

also actions by central banks, in the form of exceptional measures concerning bank liquidity and States.

The obvious response to the health crisis was confining the population in many countries [9]. This has therefore sharply reduced economic activity [10], weighed on employment, but also on the financial situation of companies [11]. Certain sectors were more particularly affected (trade, catering, tourism, construction, etc.), which required direct aid (budgetary subsidies) or indirect aid (guaranteed loans, liquidity aid), which were implemented by the monetary authorities (Central Banks). The response of governments, but also of Central banks, has led to the parenthesis of some of the regulations or laws in force, as was the case within the framework of the European Union. $^{3}$

The French government has, in particular, mobilized "exceptional powers" in times of crisis [12]. In particular it implemented a "state of health emergency" [13], based on Emmanuel Macron's speech affirming the government's total commitment and establishing "whatever the cost" as a political line, a position which was assumed from the end of March 2020 to May 2021. But, how could we evaluate this action?

This question is particularly important if we want to understand the residual weight of ideologies and practices in budgetary policies and central bank action. This allows us to understand how, in the face of an unexpected event, "powers in place" react and to what extent they remain, if not prisoners, at least influenced by the ideology and conceptions of the past.

\footnotetext{
${ }^{3}$ https://www.vie-publique.fr/en-bref/274003-covid-19-lesreponses-economiques-de-lunion-europeenne-en-debat.
} 
Table 2. Characteristics of trends

\begin{tabular}{l|c|c|c}
\hline & Budget support & Liquidity support & Total support \\
\hline (H1) & $y=-0.3742 x+0.057$ & $y=-1.2101 x+0.0339$ & $y=-1.5843 x+0.0908$ \\
R2 & 0.078 & 0.227 & 0.249 \\
(H2) & $y=0.0386 \mathrm{e}^{-8.892 x}$ & $y=0.0199 \mathrm{e}^{-20.84 x}$ & $y=0.0641 \mathrm{e}^{-14.36 x}$ \\
R2 & 0.180 & 0.463 & 0.403 \\
\hline
\end{tabular}

Various reports from the Senate [14], the Ministry of Finance [15] but also from private organizations, have tried this [16]. However, these attempts at evaluation were made in a hurry. Today, with the publication of data from the International Monetary Fund (end of January 2021 for data on support measures and April 2021 for provisional economic data [17]), it is possible to attempt a first international comparison.

The reasoning framework. Studies, and in particular those of the IMF, have shown the weight, calculated as a percentage of GDP, of aid and support to the economy, whether budgetary aid or aid to the liquidity of companies and banks. This allows for a first classification (Fig. 1).

We then see that four countries are between 30 and $45 \%$ of GDP (Japan, Italy, Germany and United Kingdom), five other countries are between 15 and 25\% (France, United States, Canada, Spain, Australia), and that the other countries range between $15 \%$ and less than $5 \%$ for the total level of aid. But, this first ranking is unsatisfactory in that it does not take into account the impact of Covid-19 on the economies, which required the support measures. However, this impact is very different depending on the economy.

This dissatisfaction leads to a search for alternative calculation methods [18]. They involve the definition of a database and the clarification of the assumptions that will be retained.
We therefore constitute a base of 25 countries from IMF data, on which there may be relative guarantees of robustness for the data for the year 2020 (Table 1). In this group of 25 countries have been included the main countries of the Euro zone and from the European Union, Russia, the United States and Canada, Australia, Japan and South Korea, but also China, India and Indonesia. For technical reasons, Latin American countries such as Argentina, Brazil and Mexico have not been included. If, the data improves, they could be included in a subsequent wave of this work as well as countries in the Middle East and Africa.

We then see that four countries are between 30 and $45 \%$ of GDP (Japan, Italy, Germany and United Kingdom), five other countries are between 15 and 25\% (France, United States, Canada, Spain, Australia), and that the other countries range between $15 \%$ and less than $5 \%$ for the total level of aid. But, this first ranking is unsatisfactory in that it does not take into account the impact of Covid-19 on the economies, which required the support measures. However, this impact is very different depending on the economy.

These data therefore include:

- The change in GDP for 2020, marked by a more or less sharp fall depending on the countries concerned, the gap ranging from $+2.9 \%$ for Vietnam to $-11 \%$ for Spain.

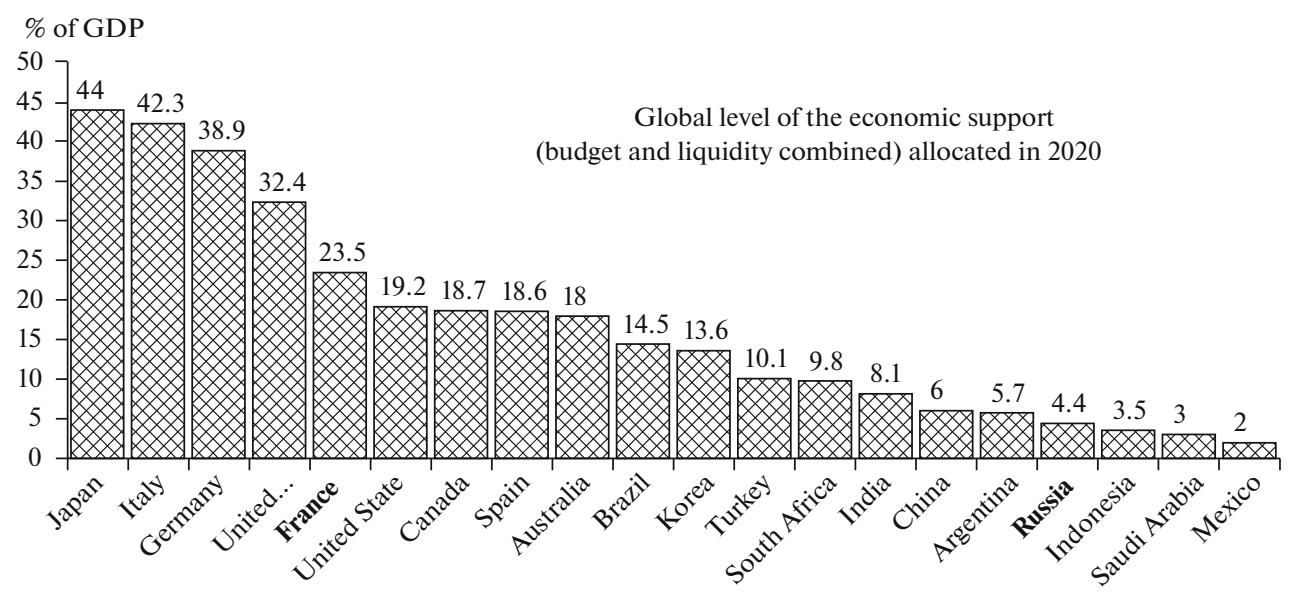

Fig. 1. Ranking according to the scale of aid to the economy. Source: IMF, IMF-FiscalDataBase, Jan. 2021 up. 
Table 3.

\begin{tabular}{|c|c|c|c|c|}
\hline \multicolumn{5}{|c|}{ Budget support (H1) } \\
\hline & $\begin{array}{l}\text { GDP changes } \\
\text { in } 2020\end{array}$ & $\begin{array}{l}\text { budget support } \\
\text { in GDP \% }\end{array}$ & $\begin{array}{l}\text { forecasted results } \\
\text { in GDP \% }\end{array}$ & $\begin{array}{l}\text { gap between actual } \\
\text { and forecasted results }\end{array}$ \\
\hline Austria & $-6.6 \%$ & $8.6 \%$ & $8.17 \%$ & $4.8 \%$ \\
\hline Belgium & $-6.4 \%$ & $7.2 \%$ & $8.10 \%$ & $-10.9 \%$ \\
\hline Finland & $-2.9 \%$ & $3.0 \%$ & $6.78 \%$ & $-56.2 \%$ \\
\hline France & $-8.2 \%$ & $7.7 \%$ & $8.78 \%$ & $-12.2 \%$ \\
\hline Germany & $-4.9 \%$ & $11.0 \%$ & $7.53 \%$ & $46.4 \%$ \\
\hline Greece & $-8.2 \%$ & $11.0 \%$ & $8.79 \%$ & $25.5 \%$ \\
\hline Ireland & $2.5 \%$ & $5.4 \%$ & $4.77 \%$ & $12.9 \%$ \\
\hline Italy & $-8.9 \%$ & $6.8 \%$ & $9.02 \%$ & $-24.3 \%$ \\
\hline Netherlands & $-3.8 \%$ & $4.5 \%$ & $7.12 \%$ & $-36.5 \%$ \\
\hline Portugal & $-7.6 \%$ & $4.7 \%$ & $8.54 \%$ & $-44.7 \%$ \\
\hline Slovakia & $-5.2 \%$ & $3.8 \%$ & $7.65 \%$ & $-50.4 \%$ \\
\hline Slovenia & $-5.5 \%$ & $5.3 \%$ & $7.77 \%$ & $-31.2 \%$ \\
\hline Spain & $-11.0 \%$ & $4.1 \%$ & $9.80 \%$ & $-57.9 \%$ \\
\hline Canada & $-5.4 \%$ & $14.6 \%$ & $7.72 \%$ & $89.7 \%$ \\
\hline Japan & $-4.8 \%$ & $15.6 \%$ & $7.51 \%$ & $107.5 \%$ \\
\hline United Kingdom & $-9.9 \%$ & $16.3 \%$ & $9.41 \%$ & $72.7 \%$ \\
\hline United States & $-3.5 \%$ & $16.7 \%$ & $7.01 \%$ & $138.5 \%$ \\
\hline Russia & $-3.1 \%$ & $2.9 \%$ & $6.86 \%$ & $-57.6 \%$ \\
\hline China & $2.3 \%$ & $4.7 \%$ & $4.85 \%$ & $-2.4 \%$ \\
\hline India & $-8.0 \%$ & $3.1 \%$ & $8.68 \%$ & $-64.8 \%$ \\
\hline Indonesia & $-2.1 \%$ & $2.7 \%$ & $6.47 \%$ & $-58.6 \%$ \\
\hline Vietnam & $2.9 \%$ & $0.5 \%$ & $4.61 \%$ & $-88.4 \%$ \\
\hline Australia & $-2.4 \%$ & $16.2 \%$ & $6.61 \%$ & $144.7 \%$ \\
\hline South Korea & $-1.0 \%$ & $3.4 \%$ & $6.06 \%$ & $-43.2 \%$ \\
\hline Sweden & $-2.8 \%$ & $5.3 \%$ & $6.75 \%$ & $-21.7 \%$ \\
\hline
\end{tabular}

Sources: IMF data for GDP and budget support. Author computation for the two others column. Data for the United States doesn't include the Biden's plan.

\begin{tabular}{|c|c|c|c|c|}
\hline \multicolumn{5}{|c|}{ Budget support (H2) } \\
\hline & $\begin{array}{l}\text { GDP changes } \\
\text { in } 2020\end{array}$ & $\begin{array}{l}\text { budget support } \\
\text { in GDP \% }\end{array}$ & $\begin{array}{l}\text { forecasted results } \\
\text { in GDP \% }\end{array}$ & $\begin{array}{l}\text { gap between actual } \\
\text { and forecasted results }\end{array}$ \\
\hline Austria & $-6.6 \%$ & $8.6 \%$ & $6.9 \%$ & $23.3 \%$ \\
\hline Belgium & $-6.4 \%$ & $7.2 \%$ & $6.8 \%$ & $5.7 \%$ \\
\hline Finland & $-2.9 \%$ & $3.0 \%$ & $5.0 \%$ & $-40.4 \%$ \\
\hline France & $-8.2 \%$ & $7.7 \%$ & $8.0 \%$ & $-3.9 \%$ \\
\hline Germany & $-4.9 \%$ & $11.0 \%$ & $6.0 \%$ & $84.8 \%$ \\
\hline Greece & $-8.2 \%$ & $11.0 \%$ & $8.0 \%$ & $37.2 \%$ \\
\hline Ireland & $2.5 \%$ & $5.4 \%$ & $3.1 \%$ & $73.9 \%$ \\
\hline Italy & $-8.9 \%$ & $6.8 \%$ & $8.5 \%$ & $-19.7 \%$ \\
\hline Netherlands & $-3.8 \%$ & $4.5 \%$ & $5.4 \%$ & $-16.5 \%$ \\
\hline Portugal & $-7.6 \%$ & $4.7 \%$ & $7.6 \%$ & $-37.6 \%$ \\
\hline Slovakia & $-5.2 \%$ & $3.8 \%$ & $6.1 \%$ & $-38.1 \%$ \\
\hline Slovenia & $-5.5 \%$ & $5.3 \%$ & $6.3 \%$ & $-15.3 \%$ \\
\hline Spain & $-11.0 \%$ & $4.1 \%$ & $10.2 \%$ & $-59.6 \%$ \\
\hline Canada & $-5.4 \%$ & $14.6 \%$ & $6.2 \%$ & $134.7 \%$ \\
\hline Japan & $-4.8 \%$ & $15.6 \%$ & $5.9 \%$ & $162.7 \%$ \\
\hline United Kingdom & $-9.9 \%$ & $16.3 \%$ & $9.3 \%$ & $74.3 \%$ \\
\hline United States & $-3.5 \%$ & $16.7 \%$ & $5.3 \%$ & $217.2 \%$ \\
\hline Russia & $-3.1 \%$ & $2.9 \%$ & $5.1 \%$ & $-42.8 \%$ \\
\hline China & $2.3 \%$ & $4.7 \%$ & $3.2 \%$ & $50.1 \%$ \\
\hline India & $-8.0 \%$ & $3.1 \%$ & $7.8 \%$ & $-61.0 \%$ \\
\hline Indonesia & $-2.1 \%$ & $2.7 \%$ & $4.6 \%$ & $-42.2 \%$ \\
\hline Vietnam & $2.9 \%$ & $0.5 \%$ & $3.0 \%$ & $-82.0 \%$ \\
\hline Australia & $-2.4 \%$ & $16.2 \%$ & $4.8 \%$ & $237.4 \%$ \\
\hline South Korea & $-1.0 \%$ & $3.4 \%$ & $4.2 \%$ & $-18.1 \%$ \\
\hline Sweden & $-2.8 \%$ & $5.3 \%$ & $5.0 \%$ & $6.6 \%$ \\
\hline
\end{tabular}

Source. Idem than in Table 3 (H1). 
Table 4.

\begin{tabular}{|c|c|c|c|c|}
\hline \multicolumn{5}{|c|}{ Liquidity support (H1) } \\
\hline & $\begin{array}{l}\text { GDP changes } \\
\text { in } 2020\end{array}$ & $\begin{array}{l}\text { liquidity support } \\
\text { in GDP \% }\end{array}$ & $\begin{array}{c}\text { forecasted results } \\
\text { in GDP \% }\end{array}$ & $\begin{array}{l}\text { gap between actual } \\
\text { and forecasted results }\end{array}$ \\
\hline Austria & $-6.6 \%$ & $2.4 \%$ & $11.4 \%$ & $-78.8 \%$ \\
\hline Belgium & $-6.4 \%$ & $11.9 \%$ & $11.2 \%$ & $6.8 \%$ \\
\hline Finland & $-2.9 \%$ & $7.0 \%$ & $6.9 \%$ & $1.3 \%$ \\
\hline France & $-8.2 \%$ & $15.8 \%$ & $13.4 \%$ & $18.0 \%$ \\
\hline Germany & $-4.9 \%$ & $27.8 \%$ & $9.3 \%$ & $198.7 \%$ \\
\hline Greece & $-8.2 \%$ & $4.9 \%$ & $13.4 \%$ & $-63.7 \%$ \\
\hline Ireland & $2.5 \%$ & $1.5 \%$ & $0.4 \%$ & $274.7 \%$ \\
\hline Italy & $-8.9 \%$ & $35.5 \%$ & $14.1 \%$ & $151.2 \%$ \\
\hline Netherlands & $-3.8 \%$ & $8.3 \%$ & $8.0 \%$ & $3.4 \%$ \\
\hline Portugal & $-7.6 \%$ & $6.5 \%$ & $12.6 \%$ & $-48.6 \%$ \\
\hline Slovakia & $-5.2 \%$ & $4.4 \%$ & $9.7 \%$ & $-54.2 \%$ \\
\hline Slovenia & $-5.5 \%$ & $6.6 \%$ & $10.1 \%$ & $-34.6 \%$ \\
\hline Spain & $-11.0 \%$ & $14.4 \%$ & $16.7 \%$ & $-13.3 \%$ \\
\hline Canada & $-5.4 \%$ & $4.0 \%$ & $9.9 \%$ & $-59.5 \%$ \\
\hline Japan & $-4.8 \%$ & $28.4 \%$ & $9.2 \%$ & $208.0 \%$ \\
\hline United Kingdom & $-9.9 \%$ & $16.1 \%$ & $15.4 \%$ & $4.7 \%$ \\
\hline United States & $-3.5 \%$ & $2.4 \%$ & $7.6 \%$ & $-68.1 \%$ \\
\hline Russia & $-3.1 \%$ & $1.5 \%$ & $7.1 \%$ & $-78.8 \%$ \\
\hline China & $2.3 \%$ & $1.3 \%$ & $0.6 \%$ & $99.9 \%$ \\
\hline India & $-8.0 \%$ & $5.1 \%$ & $13.0 \%$ & $-60.9 \%$ \\
\hline Indonesia & $-2.1 \%$ & $0.9 \%$ & $5.9 \%$ & $-85.5 \%$ \\
\hline Vietnam & $2.9 \%$ & $0.5 \%$ & $-0.1 \%$ & $-472.3 \%$ \\
\hline Australia & $-2.4 \%$ & $1.8 \%$ & $6.3 \%$ & $-71.8 \%$ \\
\hline South Korea & $-1.0 \%$ & $10.2 \%$ & $4.5 \%$ & $123.6 \%$ \\
\hline Sweden & $-2.8 \%$ & $4.2 \%$ & $6.8 \%$ & $-37.6 \%$ \\
\hline
\end{tabular}

Sources. IMF data for GDP and liquidity support. Author's computation for the two other columns.

\begin{tabular}{l|c|c|c|c}
\hline \multicolumn{5}{c}{ Liquidity support (H2) } \\
\hline & $\begin{array}{c}\text { GDP changes } \\
\text { in 2020 }\end{array}$ & $\begin{array}{c}\text { liquidity support } \\
\text { in GDP \% }\end{array}$ & $\begin{array}{c}\text { forecasted results } \\
\text { in GDP \% }\end{array}$ & $\begin{array}{c}\text { gap between actual } \\
\text { and forecasted results }\end{array}$ \\
\hline Austria & $-6.6 \%$ & $2.4 \%$ & $7.9 \%$ & $-69.4 \%$ \\
Belgium & $-6.4 \%$ & $11.9 \%$ & $7.6 \%$ & $57.1 \%$ \\
Finland & $-2.9 \%$ & $7.0 \%$ & $3.6 \%$ & $92.0 \%$ \\
France & $-\mathbf{8 . 2 \%}$ & $\mathbf{1 5 . 8 \%}$ & $\mathbf{1 1 . 1 \%}$ & $\mathbf{4 2 . 4 \%}$ \\
Germany & $-4.9 \%$ & $27.8 \%$ & $5.5 \%$ & $403.7 \%$ \\
Greece & $-8.2 \%$ & $4.9 \%$ & $11.1 \%$ & $-56.3 \%$ \\
Ireland & $2.5 \%$ & $1.5 \%$ & $1.2 \%$ & $24.6 \%$ \\
Italy & $-8.9 \%$ & $35.5 \%$ & $12.6 \%$ & $8.7 \%$ \\
Netherlands & $-3.8 \%$ & $8.3 \%$ & $9.4 \%$ & $-33.1 \%$ \\
Portugal & $-7.6 \%$ & $6.5 \%$ & $9.7 \%$ & $-24.6 \%$ \\
Slovakia & $-5.2 \%$ & $4.4 \%$ & $5.9 \%$ & $4.7 \%$ \\
Slovenia & $-5.5 \%$ & $6.6 \%$ & $19.6 \%$ & $-26.1 \%$ \\
Spain & $-11.0 \%$ & $14.4 \%$ & $6.1 \%$ & $-34.5 \%$ \\
Canada & $-5.4 \%$ & $4.0 \%$ & $5.4 \%$ & $2.3 \%$ \\
Japan & $-4.8 \%$ & $28.4 \%$ & $15.7 \%$ & $-41.1 \%$ \\
United Kingdom & $-9.9 \%$ & $16.1 \%$ & $4.1 \%$ & $-\mathbf{6 0 . 1 \%}$ \\
United States & $-3.5 \%$ & $2.4 \%$ & $3.8 \%$ & $3.6 \%$ \\
Russia & $-\mathbf{3 . 1 \%}$ & $\mathbf{1 . 5 \%}$ & $1.2 \%$ & $-51.3 \%$ \\
China & $2.3 \%$ & $1.3 \%$ & $10.5 \%$ & $-72.1 \%$ \\
India & $-8.0 \%$ & $5.1 \%$ & $3.1 \%$ & $-56.7 \%$ \\
Indonesia & $-2.1 \%$ & $0.9 \%$ & $1.1 \%$ & $-45.9 \%$ \\
Vietnam & $2.9 \%$ & $0.5 \%$ & $3.3 \%$ & $318 \%$ \\
Australia & $-2.4 \%$ & $1.8 \%$ & $2.4 \%$ & $18 \%$ \\
South Korea & $-1.0 \%$ & $3.6 \%$ & \\
Sweden & $-2.8 \%$ & $4.2 \%$ & &
\end{tabular}

Source. As in table 4 (H1). 


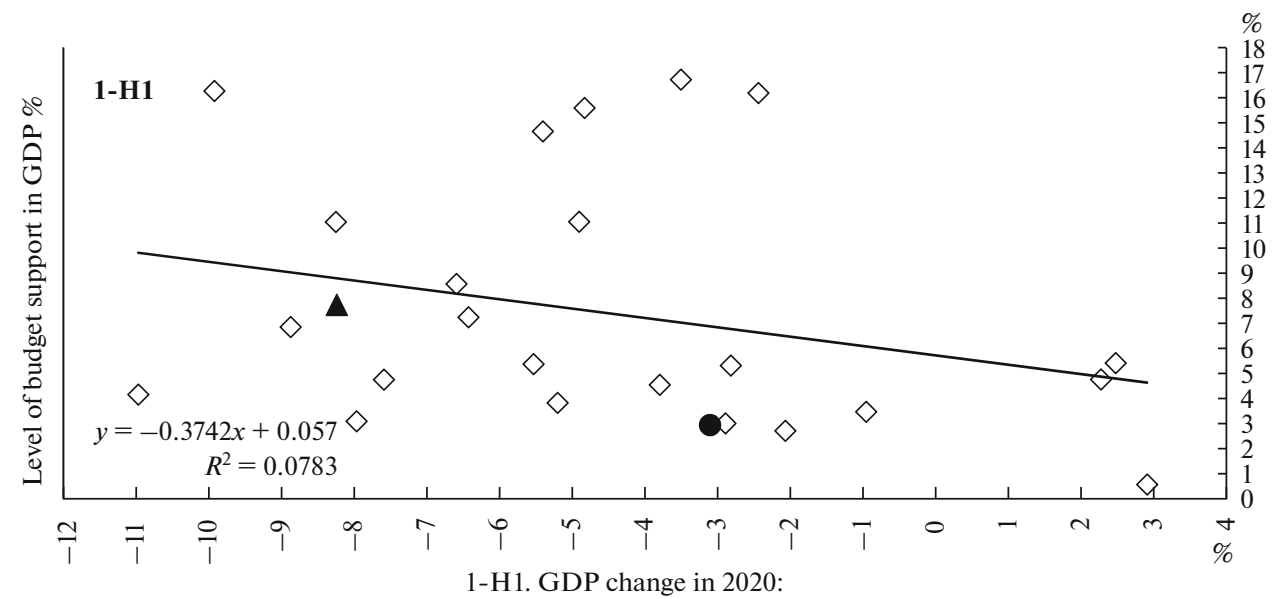

Arance;
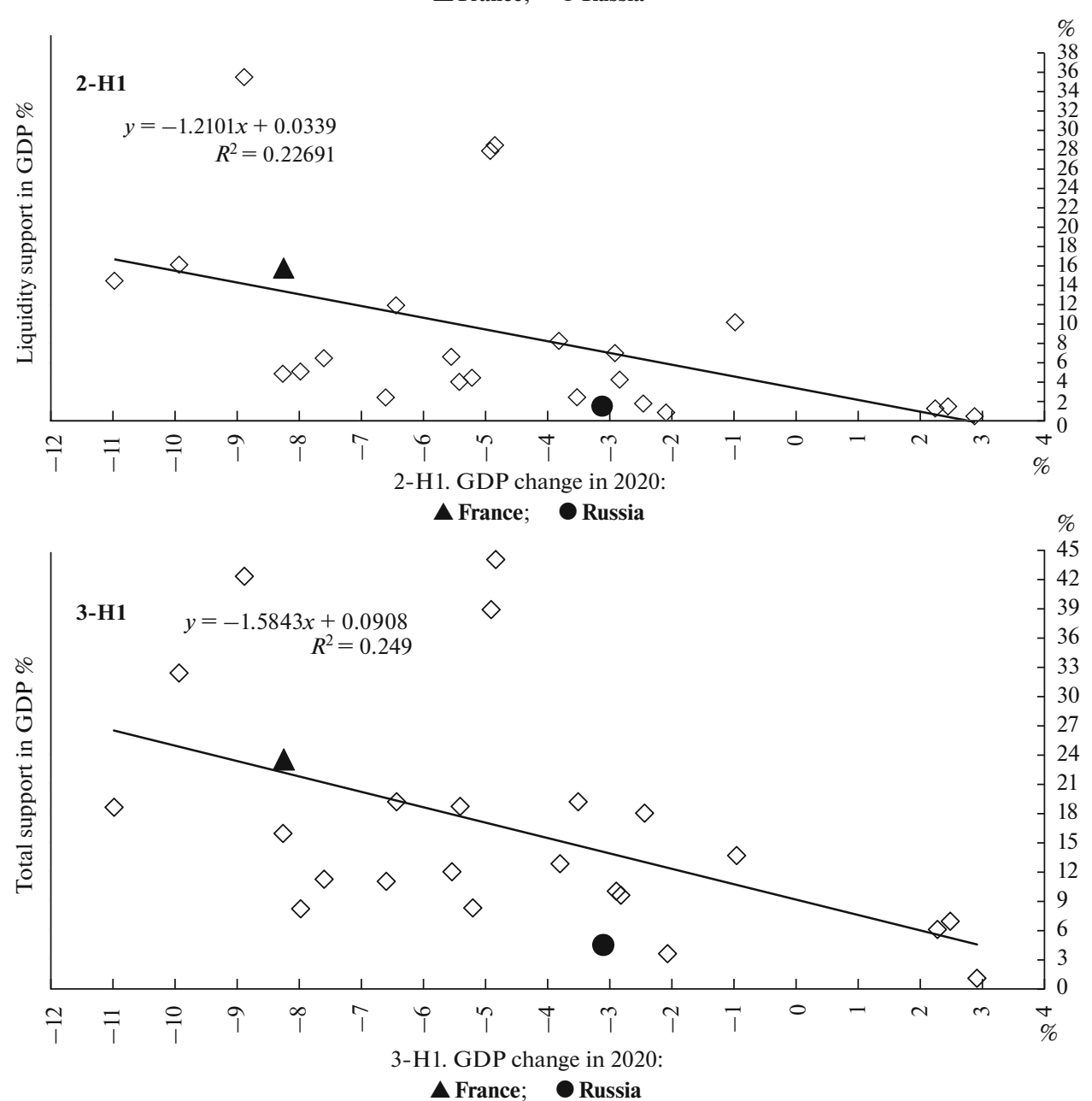

Fig. 2. Correlation between the scale of economic recession and the scale of government support. GDP change in 2020.

\section{GDP).}

- The amount of budgetary aid (as a percentage of

- The amount of discretionary liquidity aid (as a percentage of GDP).

-The sum of two previous data.
In order to be able to establish a comparison, the following assumptions and method were used:

It is assumed that the amount of aid is the result of changes in GDP, affected by a coefficient representing the more or less availability of governments and central banks to react to this change, depending on polit- 


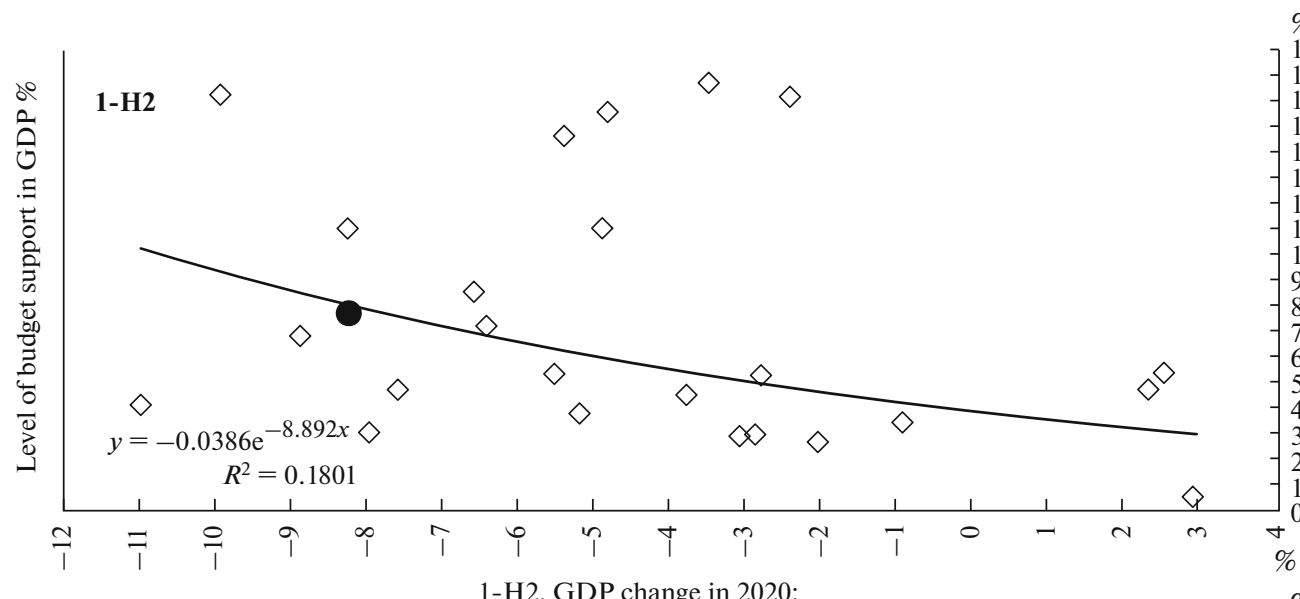

1-H2. GDP change in 2020:

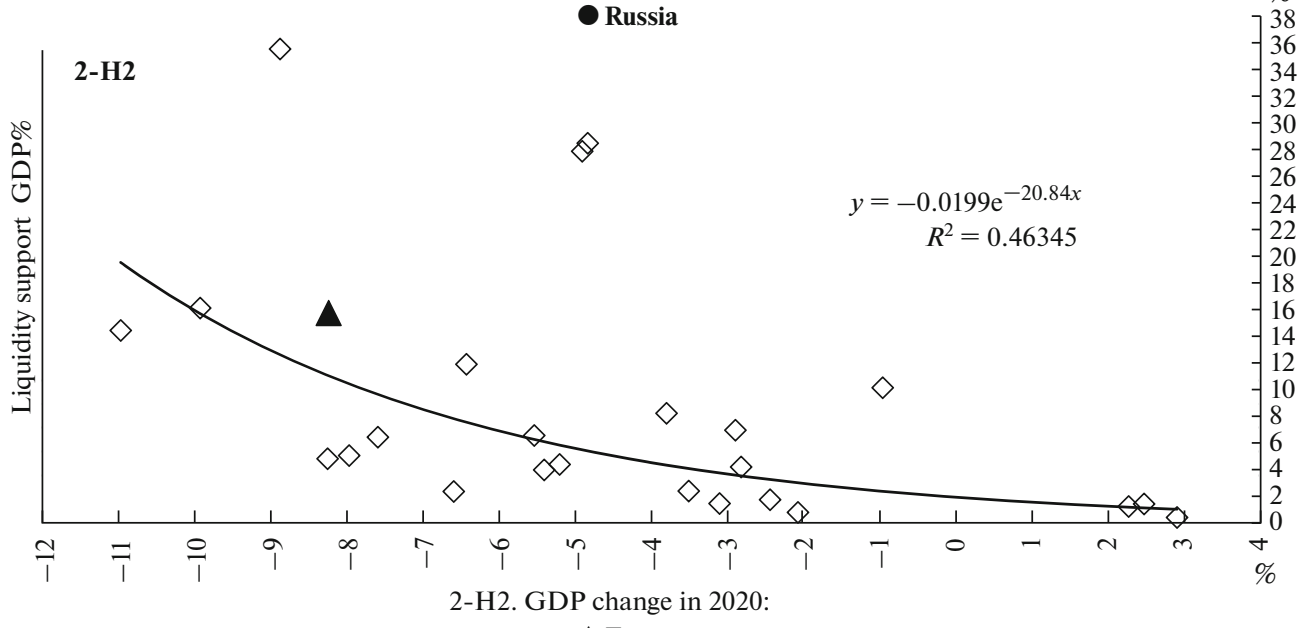

$\Delta$ France

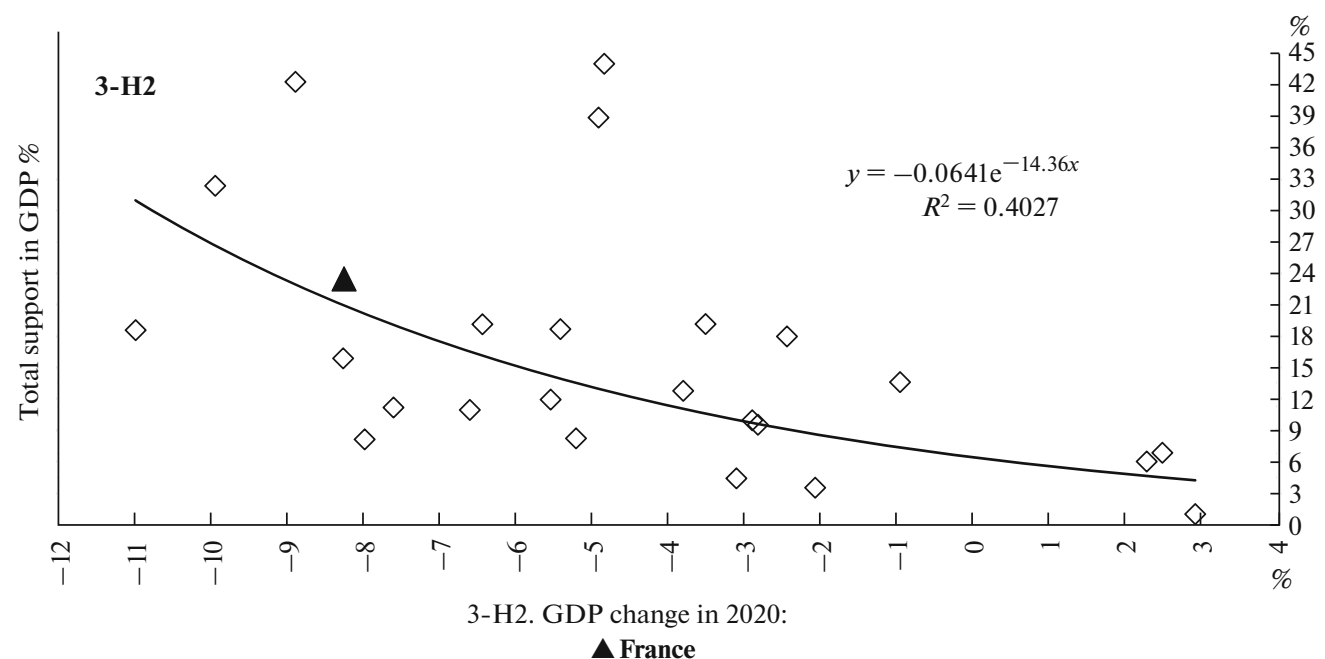

Fig. 2. (Contd.)

ical choices, but also ideological constraints specific to each country, i.e. $Y=f(x)$, with $f^{\prime}<0$ where $Y$ represents the level of aid (as a percentage of GDP), whether it is budgetary support, liquidity support or global support, and $(x)$ the evolution of GDP in 2020.
The level of economic support therefore depends on the magnitude of the shock suffered, which shock was determined by the magnitude of the health crisis and which therefore completely escapes the power of the various governments, as measured by the evolution of 
Table 5.

\begin{tabular}{|c|c|c|c|c|}
\hline \multicolumn{5}{|c|}{ Combined (budget + liquidity) total support $(\mathrm{H} 1)$} \\
\hline & $\begin{array}{l}\text { GDP changes } \\
\text { in } 2020\end{array}$ & $\begin{array}{c}\text { combined total } \\
\text { support in GDP \% }\end{array}$ & $\begin{array}{l}\text { forecasted results } \\
\text { in GDP \% }\end{array}$ & $\begin{array}{l}\text { gap between actual } \\
\text { and forecasted results }\end{array}$ \\
\hline Austria & $-6.6 \%$ & $11.0 \%$ & $19.5 \%$ & $-43.9 \%$ \\
\hline Belgium & $-6.4 \%$ & $19.1 \%$ & $19.3 \%$ & $-0.6 \%$ \\
\hline Finland & $-2.9 \%$ & $10.0 \%$ & $13.7 \%$ & $-27.1 \%$ \\
\hline France & $-8.2 \%$ & $23.5 \%$ & $22.1 \%$ & $6.1 \%$ \\
\hline Germany & $-4.9 \%$ & $38.9 \%$ & $16.8 \%$ & $130.8 \%$ \\
\hline Greece & $-8.2 \%$ & $15.9 \%$ & $22.1 \%$ & $-28.3 \%$ \\
\hline Ireland & $2.5 \%$ & $6.9 \%$ & $5.2 \%$ & $33.1 \%$ \\
\hline Italy & $-8.9 \%$ & $42.3 \%$ & $23.1 \%$ & $82.9 \%$ \\
\hline Netherlands & $-3.8 \%$ & $12.8 \%$ & $15.1 \%$ & $-15.4 \%$ \\
\hline Portugal & $-7.6 \%$ & $11.2 \%$ & $21.1 \%$ & $-47.0 \%$ \\
\hline Slovakia & $-5.2 \%$ & $8.2 \%$ & $17.3 \%$ & $-52.5 \%$ \\
\hline Slovenia & $-5.5 \%$ & $11.9 \%$ & $17.8 \%$ & $-33.1 \%$ \\
\hline Spain & $-11.0 \%$ & $18.6 \%$ & $26.5 \%$ & $-29.8 \%$ \\
\hline Canada & $-5.4 \%$ & $18.7 \%$ & $17.6 \%$ & $5.8 \%$ \\
\hline Japan & $-4.8 \%$ & $44.0 \%$ & $16.7 \%$ & $163.1 \%$ \\
\hline United Kingdom & $-9.9 \%$ & $32.4 \%$ & $24.8 \%$ & $30.6 \%$ \\
\hline United States & $-3.5 \%$ & $19.2 \%$ & $14.6 \%$ & $30.9 \%$ \\
\hline Russia & $-3.1 \%$ & $4.4 \%$ & $14.0 \%$ & $-68.4 \%$ \\
\hline China & $2.3 \%$ & $6.0 \%$ & $5.5 \%$ & $9.8 \%$ \\
\hline India & $-8.0 \%$ & $8.1 \%$ & $21.7 \%$ & $-62.4 \%$ \\
\hline Indonesia & $-2.1 \%$ & $3.5 \%$ & $12.4 \%$ & $-71.4 \%$ \\
\hline Vietnam & $2.9 \%$ & $1.0 \%$ & $4.5 \%$ & $-77.5 \%$ \\
\hline Australia & $-2.4 \%$ & $18.0 \%$ & $12.9 \%$ & $38.8 \%$ \\
\hline South Korea & $-1.0 \%$ & $13.6 \%$ & $10.6 \%$ & $28.5 \%$ \\
\hline Sweden & $-2.8 \%$ & $9.5 \%$ & $13.5 \%$ & $-29.6 \%$ \\
\hline
\end{tabular}

Combined (budget + liquidity) total support (H2)

\begin{tabular}{|c|c|c|c|c|}
\hline & $\begin{array}{l}\text { GDP changes } \\
\text { in } 2020\end{array}$ & $\begin{array}{c}\text { combined total } \\
\text { support in GDP } \%\end{array}$ & $\begin{array}{l}\text { forecasted results } \\
\text { in GDP } \%\end{array}$ & $\begin{array}{l}\text { gap between actual } \\
\text { and forecasted results }\end{array}$ \\
\hline Austria & $-6.6 \%$ & $11.0 \%$ & $16.5 \%$ & $-33.6 \%$ \\
\hline Belgium & $-6.4 \%$ & $19.1 \%$ & $16.1 \%$ & $18.7 \%$ \\
\hline Finland & $-2.9 \%$ & $10.0 \%$ & $9.7 \%$ & $2.5 \%$ \\
\hline France & $-8.2 \%$ & $23.5 \%$ & $20.9 \%$ & $12.2 \%$ \\
\hline Germany & $-4.9 \%$ & $38.9 \%$ & $13.0 \%$ & $200.0 \%$ \\
\hline Greece & $-8.2 \%$ & $15.9 \%$ & $21.0 \%$ & $-24.2 \%$ \\
\hline Ireland & $2.5 \%$ & $6.9 \%$ & $4.5 \%$ & $52.9 \%$ \\
\hline Italy & $-8.9 \%$ & $42.3 \%$ & $22.9 \%$ & $84.6 \%$ \\
\hline Netherlands & $-3.8 \%$ & $12.8 \%$ & $11.1 \%$ & $15.5 \%$ \\
\hline Portugal & $-7.6 \%$ & $11.2 \%$ & $19.1 \%$ & $-41.3 \%$ \\
\hline Slovakia & $-5.2 \%$ & $8.2 \%$ & $13.5 \%$ & $-39.1 \%$ \\
\hline Slovenia & $-5.5 \%$ & $11.9 \%$ & $14.2 \%$ & $-15.8 \%$ \\
\hline Spain & $-11.0 \%$ & $18.6 \%$ & $30.9 \%$ & $-40.0 \%$ \\
\hline Canada & $-5.4 \%$ & $18.7 \%$ & $13.9 \%$ & $34.1 \%$ \\
\hline Japan & $-4.8 \%$ & $44.0 \%$ & $12.8 \%$ & $243.2 \%$ \\
\hline United Kingdom & $-9.9 \%$ & $32.4 \%$ & $26.6 \%$ & $21.5 \%$ \\
\hline United States & $-3.5 \%$ & $19.2 \%$ & $10.6 \%$ & $80.6 \%$ \\
\hline Russia & $-3.1 \%$ & $4.4 \%$ & $10.0 \%$ & $-55.8 \%$ \\
\hline China & $2.3 \%$ & $6.0 \%$ & $4.6 \%$ & $30.1 \%$ \\
\hline India & $-8.0 \%$ & $8.1 \%$ & $20.1 \%$ & $-59.5 \%$ \\
\hline Indonesia & $-2.1 \%$ & $3.5 \%$ & $8.6 \%$ & $-59.0 \%$ \\
\hline Vietnam & $2.9 \%$ & $1.0 \%$ & $4.2 \%$ & $-76.2 \%$ \\
\hline Australia & $-2.4 \%$ & $18.0 \%$ & $9.1 \%$ & $97.5 \%$ \\
\hline South Korea & $-1.0 \%$ & $13.6 \%$ & $7.4 \%$ & $85.1 \%$ \\
\hline Sweden & $-2.8 \%$ & $9.5 \%$ & $9.6 \%$ & $-0.8 \%$ \\
\hline
\end{tabular}

Source. See Tables 3 and 4. 
Table 6.

\begin{tabular}{|c|c|c|c|c|c|c|c|c|}
\hline \multicolumn{9}{|c|}{ Ranking of countries (H1) } \\
\hline \multicolumn{3}{|c|}{ budget support, deviation to the trend } & \multicolumn{3}{|c|}{ liquidity support, deviation to the trend } & \multicolumn{3}{|c|}{ total support, deviation to the trend } \\
\hline Australia & $144.67 \%$ & 1 & Ireland & $274.70 \%$ & 1 & Japan & $163.1 \%$ & 1 \\
\hline United States & $138.46 \%$ & 2 & Japan & $207.95 \%$ & 2 & Germany & $130.8 \%$ & 2 \\
\hline Japan & $107.53 \%$ & 3 & Germany & $198.71 \%$ & 3 & Italy & $82.9 \%$ & 3 \\
\hline Canada & $89.67 \%$ & 4 & Italy & $151.19 \%$ & 4 & Australia & $38.8 \%$ & 4 \\
\hline United Kingdom & $72.73 \%$ & 5 & Korea & $123.58 \%$ & 5 & Ireland & $33.1 \%$ & 5 \\
\hline Germany & $46.44 \%$ & 6 & China & $99.86 \%$ & 6 & United States & $30.9 \%$ & 6 \\
\hline Greece & $25.52 \%$ & 7 & France & $17.99 \%$ & 7 & United Kingdom & $30.6 \%$ & 7 \\
\hline Ireland & $12.85 \%$ & 8 & Belgium & $6.79 \%$ & 8 & Korea & $28.5 \%$ & 8 \\
\hline Austria & $4.76 \%$ & 9 & United Kingdom & $4.69 \%$ & 9 & China & $9.8 \%$ & 9 \\
\hline China & $-2.38 \%$ & 10 & Netherlands & $3.36 \%$ & 10 & France & $6.1 \%$ & 10 \\
\hline Belgium & $-10.90 \%$ & 11 & Finland & $1.35 \%$ & 11 & Canada & $5.8 \%$ & 11 \\
\hline France & $-12.19 \%$ & 12 & Espagne & $-13.29 \%$ & 12 & Belgium & $-0.6 \%$ & 12 \\
\hline Sweden & $-21.71 \%$ & 13 & Slovenia & $-34.55 \%$ & 13 & Netherlands & $-15.4 \%$ & 13 \\
\hline Italy & $-24.33 \%$ & 14 & Sweden & $-37.61 \%$ & 14 & Finland & $-27.1 \%$ & 14 \\
\hline Slovenia & $-31.20 \%$ & 15 & Portugal & $-48.56 \%$ & 15 & Greece & $-28.3 \%$ & 15 \\
\hline Netherlands & $-36.53 \%$ & 16 & Slovakia & $-54.17 \%$ & 16 & Sweden & $-29.6 \%$ & 16 \\
\hline Korea & $-43.18 \%$ & 17 & Canada & $-59.50 \%$ & 17 & Spain & $-29.8 \%$ & 17 \\
\hline Portugal & $-44.66 \%$ & 18 & India & $-60.92 \%$ & 18 & Slovenia & $-33.1 \%$ & 18 \\
\hline Slovakia & $-50.36 \%$ & 19 & Greece & $-63.71 \%$ & 19 & Austria & $-43.9 \%$ & 19 \\
\hline Finland & $-56.15 \%$ & 20 & United States & $-68.11 \%$ & 20 & Portugal & $-47.0 \%$ & 20 \\
\hline Russia & $-57.59 \%$ & 21 & Australia & $-71.77 \%$ & 21 & Slovakia & $-52.5 \%$ & 21 \\
\hline Spain & $-57.87 \%$ & 22 & Russia & $-78.80 \%$ & 22 & India & $-62.4 \%$ & 22 \\
\hline Indonesia & $-58.60 \%$ & 23 & Austria & $-78.83 \%$ & 23 & Russia & $-68.4 \%$ & 23 \\
\hline India & $-64.78 \%$ & 24 & Indonesia & $-85.52 \%$ & 24 & Indonesia & $-71.4 \%$ & 24 \\
\hline Vietnam & $-88.39 \%$ & 25 & Vietnam & $-472.26 \%$ & 25 & Vietnam & $-77.5 \%$ & 25 \\
\hline \multicolumn{9}{|c|}{ Ranking of countries $(\mathrm{H} 2)$} \\
\hline \multicolumn{3}{|c|}{ budget support, deviation to the trend } & \multicolumn{3}{|c|}{ liquidity support, deviation to the trend } & \multicolumn{3}{|c|}{ total support, deviation to the trend } \\
\hline Australia & $237.4 \%$ & 1 & Japan & $422.3 \%$ & 1 & Japan & $243.2 \%$ & 1 \\
\hline United States & $217.2 \%$ & 2 & Germany & $403.7 \%$ & 2 & Germany & $200.0 \%$ & 2 \\
\hline Japan & $162.7 \%$ & 3 & Korea & $318.6 \%$ & 3 & Australia & $97.5 \%$ & 3 \\
\hline Canada & $134.7 \%$ & 4 & Italy & $180.7 \%$ & 4 & Korea & $85.1 \%$ & 4 \\
\hline Germany & $84.8 \%$ & 5 & Finland & $92.0 \%$ & 5 & Italy & $84.6 \%$ & 5 \\
\hline United Kingdom & $74.3 \%$ & 6 & Netherlands & $88.0 \%$ & 6 & United-States & $80.6 \%$ & 6 \\
\hline Ireland & $73.9 \%$ & 7 & Belgium & $57.1 \%$ & 7 & Ireland & $52.9 \%$ & 7 \\
\hline China & $50.1 \%$ & 8 & France & $42.4 \%$ & 8 & Canada & $34.1 \%$ & 8 \\
\hline Greece & $37.2 \%$ & 9 & Ireland & $24.6 \%$ & 9 & China & $30.1 \%$ & 9 \\
\hline Austria & $23.3 \%$ & 10 & Sweden & $18.5 \%$ & 10 & United Kingdom & $21.5 \%$ & 10 \\
\hline Sweden & $6.6 \%$ & 11 & Slovenia & $4.7 \%$ & 11 & Belgium & $18.7 \%$ & 11 \\
\hline Belgium & $5.7 \%$ & 12 & China & $3.6 \%$ & 12 & Netherlands & $15.5 \%$ & 12 \\
\hline France & $-3.9 \%$ & 13 & United Kingdom & $2.5 \%$ & 13 & France & $12.2 \%$ & 13 \\
\hline Slovenia & $-15.3 \%$ & 14 & Slovakia & $-24.6 \%$ & 14 & Finland & $2.5 \%$ & 14 \\
\hline Netherlands & $-16.5 \%$ & 15 & Spain & $-26.1 \%$ & 15 & Sweden & $-0.8 \%$ & 15 \\
\hline Korea & $-18.1 \%$ & 16 & Portugal & $-33.1 \%$ & 16 & Slovenia & $-15.8 \%$ & 16 \\
\hline Italy & $-19.7 \%$ & 17 & Canada & $-34.5 \%$ & 17 & Greec & $-24.2 \%$ & 17 \\
\hline Portugal & $-37.6 \%$ & 18 & United States & $-41.1 \%$ & 18 & Austria & $-33.6 \%$ & 18 \\
\hline Slovakia & $-38.1 \%$ & 19 & Australia & $-45.9 \%$ & 19 & Slovakia & $-39.1 \%$ & 19 \\
\hline Finland & $-40.4 \%$ & 20 & India & $-51.3 \%$ & 20 & Spain & $-40.0 \%$ & 20 \\
\hline Indonesia & $-42.2 \%$ & 21 & Greece & $-56.3 \%$ & 21 & Portugal & $-41.3 \%$ & 21 \\
\hline Russia & $-42.8 \%$ & 22 & Vietnam & $-56.7 \%$ & 22 & Russia & $-55.8 \%$ & 22 \\
\hline Spain & $-59.6 \%$ & 23 & Russia & $-60.1 \%$ & 23 & Indonesia & $-59.0 \%$ & 23 \\
\hline India & $-61.0 \%$ & 24 & Austria & $-69.4 \%$ & 24 & India & $-59.5 \%$ & 24 \\
\hline Vietnam & $-82.0 \%$ & 25 & Indonesia & $-72.1 \%$ & 25 & Vietnam & $-76.2 \%$ & 25 \\
\hline
\end{tabular}


GDP for the year 2020. As for the impact of these support measures, it could ONLY be measured on the GDP of 2021.

This function, $Y=f(x)$, is calculated either in a linear hypothesis now called H1 $(Y=a(x)+b)$, which implies that the reaction is directly in proportion to the shock or in an exponential hypothesis, now called $\mathrm{H} 2$ $(Y=a(E-(x))$, which assumes that the greater the shock, the more the authorities' reaction to a non-proportional extent. here estimated on the available data.

The meaning of these two hypotheses is therefore as follows. In (H1) it is assumed that the depth of the recession experienced in 2020 generates a support response lineal to the recession: the greater the recession, the more the support. In hypothesis (H2) we assume that the support is more than proportional to the depth of the recession and that a deepening of the recession forces the authorities of the country to increase their support to the economy much more.

We represent the potential relationship for the three data (budget aid, liquidity aid and total aid) in the form of a point cloud from which we extract a trend line. $Y$ is then calculated, which is the theoretical value of the aid obtained from the calculation of the fit of the data.

The deviation from the theoretical value of real aid is determined for each country by the formula $Z=Y-Y^{r}$ and the countries are ranked from the highest value to the lowest value. We will therefore not compare the direct figures of the support measures of each country, as we have done in previous publications [19], but the extent of the gap between the predicted value of the support and its actual value.

It is important to stress here that the amount of aid is decided either by the government (budgetary aid) or by the monetary authorities (central banks) which, depending on their degree of independence from political power, have a more reactive function. or less distinct from the latter.

Let us point out here some reservations about the available data.

If the amount of liquidity support by the Central Bank of China is known, it cannot be excluded that local authorities had initiated other support measures. The same reasoning also applies to budgetary support. The aid figure therefore represents a "minimum" figure.

This reasoning may apply, to a lesser extent, to another federal country of the sample, which is India.

The GDP figures correspond to the estimates of the statistical services as they were at the beginning of the year. It is not impossible that the final figures, which should be known in 2023, are slightly different from the figures, which are used in this study.

The characteristics of the calculated trends are then as follows (Table 2).

The levels of the correlation coefficients are low, but appear a little better when the calculation is made for the total support (budgetary and in liquidity). This is not completely surprising. Correlation coefficients appear too to be better in the $(\mathrm{H} 2)$ hypothesis than in (H1). This too was expected (see Fig. 2).

Indeed one could expect that the reactions of each country would be specific, depending, as has been said, on a mixture of ideological assumptions but also political and economic constraints. The dispersion from the alignment curve is therefore not surprising.

Likewise, the relative improvement in results with the addition of forms of aid and support indicates to us, even before any in-depth analysis that certain countries have "compensated" for a lower budgetary effort by a greater commitment in liquidity and -vice versa.

\section{Several remarks emerge then.}

On the one hand, the adjustment to an exponential trend $(\mathrm{H} 2)$ seems to be more realistic than that to a linear trend, thus validating the hypothesis that, all other things being equal (political and ideological bias), the support function was not a linear one. The more the recession was strong and more the effort was more than proportional to the recession. Then, on the other hand, with regard to budgetary aid, a group of four countries stand out both by the moderate amount of the recession they have suffered and by the high amount of budgetary aid: Australia, Canada, Japan and the United States. This group is interesting because it combines two islands (Japan and Australia) and two mainland countries (Canada and United States), two countries with low population density (Australia and Canada), one medium density country (United States) and one very high-density country (Japan) (Table 3).

In the hypothesis (H1) (Table 3) the mean deviation is at $-0.7 \%$ while it is $+25 \%$ in (H2) (Table 3 ). Clearly, the apparently more realistic hypothesis (H2) tends to push up the result for some countries. If the amount of support is comparable to the United Kingdom, the recession suffered is, for its part, much more considerable. Likewise, the situations of China and Ireland emerge. For both economies the economic results, by comparison, appear good, and the level of budget support is relatively high by comparison with the recession shock, but which are, in all respects, very dissimilar. France is here in 12th place.

In the hypothesis $(\mathrm{H} 2)$, the average deviation is $25 \%$ and the group of countries showing the deviation from the largest trend includes only non-European countries (Australia, United States, Japan, Canada), then followed by a group of countries where China and the United Kingdom figure prominently. France, in 13th position, is therefore below the median. The adoption of $(\mathrm{H} 2)$, while improving the overall result, results in a deterioration of France's position.

With regard to liquidity aid, the same phenomenon can be observed: the average difference, which was 
Table 7. Distribution of deviations from the trend in hypothesis $\mathrm{H} 2$ (the "+" sign indicates a particularly important effort (more than 100\%) and the sign-an effort well below the trend (-55\%), quotations for budgetary aid and parenthesis for liquidity aid

\begin{tabular}{|c|c|c|}
\hline & $\begin{array}{l}\text { Weak budget support } \\
\text { (negative deviation to the trend) }\end{array}$ & $\begin{array}{c}\text { Strong budget support } \\
\text { (positive deviation to the trend) }\end{array}$ \\
\hline $\begin{array}{l}\text { Strong support form the Central Bank } \\
\text { (positive deviation to the trend) }\end{array}$ & $\begin{array}{l}\text { Korea "+” } \\
\text { Italy “+” } \\
\text { Finland } \\
\text { France } \\
\text { Netherlands } \\
\text { Slovenia }\end{array}$ & $\begin{array}{l}\text { Japan “+” “+” } \\
\text { Germany “+” } \\
\text { Ireland "+" } \\
\text { United Kingdom } \\
\text { Sweden } \\
\text { Belgium } \\
\text { China }\end{array}$ \\
\hline $\begin{array}{l}\text { Weak support from the Central Bank } \\
\text { (negative deviation to the trend) }\end{array}$ & $\begin{array}{c}\text { Vietnam “-” }(-) \\
\text { Slovakia } \\
\text { Russia (-) } \\
\text { Portugal } \\
\text { Spain (-) } \\
\text { Indonesia } \\
\text { India (-) }\end{array}$ & $\begin{array}{c}\text { Australia “+” } \\
\text { United States "+" } \\
\text { Canada “+” } \\
\text { Austria (-) } \\
\text { Greece (-) }\end{array}$ \\
\hline
\end{tabular}

$-5.5 \%$ with (H1) (Table 4) increases to $+43 \%$ with (H2) (Table 4).

Three countries, under the hypothesis $(\mathrm{H} 1)$ stand out from the whole with a deviation from the trend in the amount of aid greater than $200 \%$ of GDP, Ireland and Japan, while Germany, Italy and Korea have a deviation between 100 and $200 \%$. These are five highly industrialized economies, including three countries in the EMU. The role of the ECB is well highlighted here, while the EU and EMU countries appear to be much less well placed for budgetary aid. In hypothesis $(\mathrm{H} 2)$, three countries show a deviation from the trend of more than $300 \%$, Germany, Japan, and South Korea. They are followed by four other EMU countries: Italy, Finland, the Netherlands and Belgium. The latter moves back 4 places from its position in hypothesis (H1). It should be noted that the sample seems fairly evenly distributed, even if the group of the 4 leading countries tends to unbalance it. Conversely, five countries have very large gaps in liquidity aid in the negative direction: Australia, Austria, Russia, Indonesia and Vietnam in the hypothesis (H1).

France has a difference equal to the average difference in $(\mathrm{H} 2)$ while its difference was greatly greater than the average difference in $(\mathrm{H} 1)$, proof that the adoption of $(\mathrm{H} 2)$, if it improves overall the result on 1 'sample degrades that of France. She also went from 7 th place in $(\mathrm{H} 1)$ to 8 th place in $(\mathrm{H} 2)$. Moreover, in $(\mathrm{H} 2)$ the group of countries with the deviation from the worst trend is a little different from what we had observed in (H1), since it includes Greece, India, Vietnam, Russia, Austria and Indonesia. In (H2), 13 countries show a deviation from the positive trend.

The analysis of liquidity aid also reveals important points.
Support measures for households, businesses and the financial sector have therefore focused both on liquidity (with the supply of credit and the deferral of financial obligations) and on their solvency (with the transfer of real resources). This has forced governments to make several arbitrations. If transfers or soft loans were made to a large corporation, they may have been made conditional on continued employment and limited executive compensation, or dividends and share buybacks. An intermediate option was for the state to take a stake in the company. Where liquidity was a major problem, recourse was had to central bank credit (through an asset purchase program) or other state-controlled financial intermediaries (through loans and guarantees).

Such practices had proven effective in previous crises. We can, then, compare the deviations to the trend for the total support to the economy in hypothesis (H1) (Table 5) as in hypothesis (H2) (Table 5).

The change from $(\mathrm{H} 1)$ to $(\mathrm{H} 2)$ then has notable effects. First, the average deviation from the trend goes from $-1 \%$ to $+21 \%$. Then, if the group of countries with the largest positive deviation has only 3 countries, Japan, Germany and Italy, this group is significantly larger, but split in two in (H2) with Japan and Italy and Germany in the lead (gap of more than 200\%) followed by 4 other countries, Australia, South Korea, Italy and the United States.

The combination of budgetary aid and liquidity aid naturally implies that the extra-EU countries, and extra-European countries in general, rise massively in the rankings. We can therefore see that there is a peculiarity of EU countries, which have, in general, given less budget support to their economies than non-EU countries. On the other hand, the countries of the EMU, in other words of the euro zone, benefit from 
Table 8. Ranking of Euro zone countries according to public debt and the deviation from the trend in budgetary aid $(\mathrm{H} 2)$

\begin{tabular}{l|c|c}
\hline & $\begin{array}{c}\text { Public debt } \\
\text { in 2019 (GDP \%) }\end{array}$ & $\begin{array}{c}\text { Budget support, deviation } \\
\text { to the trend (H2) }\end{array}$ \\
\hline Netherlands & $47.6 \%$ & $-16.5 \%$ \\
Slovakia & $48.5 \%$ & $-38.1 \%$ \\
Ireland & $57.4 \%$ & $73.9 \%$ \\
Finland & $59.3 \%$ & $-40.4 \%$ \\
Germany & $59.6 \%$ & $84.8 \%$ \\
Slovenia & $65.6 \%$ & $-15.3 \%$ \\
Austria & $70.5 \%$ & $23.3 \%$ \\
Spain & $95.5 \%$ & $-59.6 \%$ \\
Belgium & $98.1 \%$ & $5.7 \%$ \\
France & $\mathbf{9 8 . 1 \%}$ & $-3.9 \%$ \\
Portugal & $116.8 \%$ & $-37.6 \%$ \\
Italy & $134.6 \%$ & $-19.7 \%$ \\
Greece & $184.9 \%$ & $37.2 \%$ \\
\hline
\end{tabular}

Source: FMI.

overall greater liquidity aid, which explains their rise in the ranking. However, the ECB's contribution is generally insufficient to enable them to improve their position in the overall ranking.

France, meanwhile, moved from 10th place in (H1) (Table 6) to 13th place in (H2) (Table 6). Generally speaking, the use of an exponential trend versus a linear one improves the reliability of comparisons. However, the exponential trend $(\mathrm{H} 2)$, because it makes it possible to take better account of the results at the extremes, both better highlights the efforts made by the countries where the recession was relatively weak, and in return shows the widening of the results for countries in the middle of the sample.

Results analysis. How can the differences between various countries in terms of forms of political reaction, economic ideology, or both economic and institutional constraints explain the differences in reaction to the Covid-19 pandemic and the differences in the level of support for the economy [20]? This question can then be approached now more rationally.

The first thing that strikes you is the diversity of the results. It also reflects the diversity of national responses to the pandemic.

The results, once expressed as a percentage of the deviation from the sample trend, thus show a clear difference between budget aid and liquidity aid. Under hypothesis ( $\mathrm{H} 1)$, for budgetary aid, only nine countries $(36 \%)$ are above the trend, including three, as already noted, very much above, with a difference of more than 100\% (Australia, United States, Japan). The latter have clearly reacted very vigorously to the economic consequences of the pandemic. They are followed by another group of three countries with deviations from the budget support trend of between 90\% and 45\%, Canada, the United Kingdom and Germany. France, despite President Emmanuel Macron's "whatever it takes", only finds itself in 12th rank, the median of the sample ahead of four other Euro zone countries: Greece, Ireland, Austria and Belgium.

As regards liquidity aid, eleven countries show a positive deviation from the trend, ie $44 \%$ of the sample. But, there is a phenomenon of net compensation in relation to budgetary aid. Six countries are in fact with deviations of $99 \%$ or more from the trend (Ireland, Japan, Germany, Italy, South Korea, China). For these countries, with the exception of Germany and Japan, there is a form of compensation. France follows immediately behind, but with a gap of only $18 \%$.

If we now observe the total of aid, we see that France is in tenth position (mainly thanks to the contribution of aid to liquidity) within the framework of (H1) and that it moves to thirteenth position in the frame of $(\mathrm{H} 2)$. Compared to the severity of the consequences of the health crisis, it is far behind both Japan and Germany (whose total difference is more than $100 \%$ in $\mathrm{H} 1$ and more than $200 \%$ in H2) and by Italy, the United Kingdom, the United States or even Ireland or South Korea and even (H2) Belgium and the Netherlands. This encourages us to put into perspective the self-satisfaction claims of our leaders and specifically of President Emmanuel Macron. France is clearly not in the leading group of countries that have helped their economies the most in the face of the consequences of the health crisis. If we can understand that it made a lesser effort than those made by Japan and Germany, Italy, Ireland and the United Kingdom made more efforts ( $\mathrm{H} 1)$, countries to which it must be added Belgium and the Netherlands (H2). In general, the EU countries, with the exception of Germany, have provided less budgetary support for their economies than the rest of the world, and the action of the ECB, however proactive it may have been, did not prove to be sufficient to redress the situation. As can be seen, monetary policy is not completely substitutable for fiscal policy (Table 7).

We can then attempt to answer some of the questions asked in the introduction.

In the group of 6 countries of the «northeast» quadrant, which corresponds to a double level of budget and liquidity support, the presence of Japan and Germany with massive levels of support compared to the level of economic shock caused by Covid-19 suggests that governments have profited from a windfall effect. With the epidemic weakening the political and ideological constraints on these two points, it cannot be ruled out that they have sought to use the pretext of the crisis to massively help their economies. This seems to be particularly true in the case of Germany, but may be less certain for Japan, where monetary and 
fiscal policy constraints are less obvious as shown by economic policy since 2012.

United Kingdom and Sweden seem to have wanted to respond to an initially inadequate health policy in order to reconcile their populations, and this seems particularly true for the "post-Brexit" United Kingdom. Finally, we have in this group China, whose level of shock due to Covid is generally very low and of the same order as in Vietnam, which is on the other hand in the "southwest" quadrant, characterized by levels very low support, both budgetary and liquidity. Is it due to the greater sensitivity of the Chinese authorities to the social movements occurring in the country? We can think so.

If we now look at the countries that have helped their economies the least ("southwest" quadrant), we find countries that have done so out of ideological or political choice (Slovakia and Russia). For Slovakia, a member country of the EU and the EMU (Euro zone), it is known that the government of this country is to very close to the group of "frugal" countries that have opposed a strong support by the ECB. He could therefore be expected to use budget support sparingly. It is nevertheless more astonishing that he made very little use of the liquidity aid allowed by the ECB.

In the case of Russia, we know the prudence of the government and of the Central Bank. It should be noted, however, that the level of budgetary aid was lower than it had been during the 2008-2009 crisis when the government had reacted quite strongly. There are also countries which, either because the consequences of the health crisis were weak (the case of Vietnam), or because their means are limited (India, Indonesia). It is to be noted that growth has been $2.9 \%$ in Vietnam, one of the less affected country, $-2.1 \%$ in Indonesia and $-3.1 \%$ in Russia. It may also explain why their respective governments found themselves faced with a lesser injunction to support their economies. But, this way of reasoning apply neither for India $(-8 \%)$ nor for Slovakia $(-5.2 \%)$.

It should also be noted that the countries of Southern Europe (Spain, Greece, Portugal) where the consequences of the debt crisis and the Euro crisis have not yet been overcome have reacted more weakly to the recession and have unwanted or were unable to use the resources made available to governments by the ECB. From this point of view, the Euro zone has widened the differences within it.

The "north-eastern" quadrant, which corresponds to a low level of budget support but a high level of liquidity aid is interesting because of the 6 countries it contains, 5 are part of the EU and EMU.

It is therefore revealing that these countries made relatively little use of the budgetary instrument, even though their situation in terms of public debt was very different (the debt of Italy and France is high but that of Finland and of the Netherlands is much weaker) while on the other hand they have massively used the liquidity instrument (Table 8).

This is indicative of the relative institutional weight of the Euro zone where some countries over-react in terms of fiscal prudence (the Netherlands, Slovakia, Finland and Slovenia) while their debt situation would have allowed them to have much more margins to manoeuver.

The health crisis thus turns out to have been a powerful revealed of the behaviours, the constraints, but also the ideological presuppositions of each other. The differences in the levels of support for the economy in the aftermath of the Covid-19 crisis provide a measure of how these different facto rs influence government responses.

\section{ACKNOWLEDGMENTS}

I'm deeply grateful to professors Kuvalin and Volkonskii for their remarks and comments on an earlier version of this text when presented to the 61st session of the French-Russian seminar.

\section{REFERENCES}

1. C. Silvestre, "The mother of all recessions has arrived," in The UniCredit Economics Chartbook (UniCredit Bank, Milan, 2020).

2. S. Ng, Modeling Macroeconomic Variations after COVID-19: Working Paper 29060 (National Bureau of Economic Research, Cambridge, MA, 2021).

3. J. Sapir, "Would the lock-down induced economic contraction be a prelude to a major depression?" Econ. Math. Methods. 56 (3), 5-25 (2020).

4. L. Fornaro and M. Wolf, Covid-19 Coronavirus and Macroeconomic Policy: BSE Working Paper 1168 (Barcelona School of Economics, Barcelona, 2020).

5. T. Hale, N. Angrist, E. Cameron-Blake, L. Hallas, B. Kira, S. Majumdar, A. Petherick, T. Phillips, H. Tatlow, and S. Webster, Oxford COVID-19 Government Response Tracker (Blavatnik School of Government, Oxford, 2020).

6. H. Herrera, M. Konradt, G. Ordoñez, and C. Trebesch, The political consequences of the Covid pandemic: Lessons from cross-country polling data. https://voxeu.org/article/political-consequences-covidpandemic.

7. M. A. Ruiz Estrada, "How much unemployment and inflation is going to be generated by COVID-19?" Transnatl. Corp. Rev. 13 (2), 202-210 (2021). https://doi.org/10.1080/19186444.2021.1912991; R. Kollmann, Effects of Covid-19 on Euro Area GDP and inflation: demand vs. supply disturbances, MPRA Paper no. 108285, 2021. https://mpra.ub.unimuenchen.de/108285/.

8. C. Cottarelli, "Euro Area fiscal policies and capacity in post-pandemic times" in Economic Governance Support Unit (European Parliament, Bruxelles, Juin 2021).

9. F. Alvarez, D. Argente, and F. Lippi, "A simple planning problem for COVID-19 lockdown, testing, and 
tracing," Am. Econ. Rev.: Insights 3 (3), 367-382 (2021).

10. J. Sapir, "Would the lock-down induced economic contraction be a prelude to a major depression?", Ekon. Matem. Metody vol. 56 (3), 5-25 (2020).

11. C. H. Ebeke, N. Jovanovic, L. Valderrama, and J. Zhou, Corporate Liquidity and Solvency in Europe during COVID- 19: The Role of Policies: IMF Working Paper 2021/056 (International Monetary Fund, Washington, DC, 2021).

12. R. Savy, "Les pouvoirs économiques exceptionnels," Pouvoirs, No. 10, (September 1979).

13. The "State of health emergency" was first implemented by the March 23, 2020 law, and was prolonged till July 10,2020 . It was again implemented from October 17, 2020 and consolidated by the November 14, 2020 law that extended first the emergency till February 16, 2021 and got renewed by the February 15, 2021 law till June 1st, 2021. The May 31, 2021 law managing the exit of the emergency has organised a provisional regime from June 2 to September 30, 2021. But, a new law, dating of August 9th, has again extended the emergency till November 15, 2021. https://www.vie-publique.fr/fiches/273947-quest-ce-que-letat-durgence-sanitaire.

14. Commission d'enquête pour l'évaluation des politiques publiques face aux grandes pandémies à la lumière de la crise sanitaire de la covid-19 et de sa gestion, Sénat de la République Française in Rapport No. 199, sous la prés dence de M. Alain Milon, 8 décembre 2020. http://www.senat.fr/commission/enquete/gestion de_la_crise_sanitaire.html.

15. Comité de Suivi et d'Évaluation des Mesures de Soutien Financier aux Entreprises Confrontées à l'Épidémie de Covid-19 in Rapport d'Étape (Inspection Générale des Finances, Paris, 2021).

16. N. Bauquet, L'action publique face à la crise du Covid-19, Institut Montaigne, 2020. https://www.institutmontaigne. org/publications/laction-publique-face-lacrise-du-covid-19.

17. World economic outlook, update, January 2021, World economic outlook, April 2021, and fiscal monitor: database of country fiscal measures in response to the COVID-19 pandemic. https://www.imf.org/.

18. A. Chudik, K. Mohaddes, M. H. Pesaran, M. Raissi, and A. Rebucci, A Counterfactual Economic Analysis of Covid-19 Using a Threshold Augmented Multi-Country Model: Working Paper 27855 (National Bureau of Economic Research, Cambridge, MA, 2020).

19. J. Sapir, "Is Eurozone accumulating an historical lag toward Asia in the Covid-19 context?" Econ. Revival Russ., No. 1 (67), 89-102 (2021).

20. S. L. Greer, E. J. King, E. M. da Fonseca, and A. Peralta-Santos, "The comparative politics of COVID-19: The need to understand government responses," Global Publ. Health 15 (9), pp. 1413-1416 (2020); A. R. Migone, "The influence of national policy characteristics on COVID-19 containment policies: a comparative analysis," Policy Des. Pract. 3, 259-276 (2020). 